\title{
Size optimization for mirror segments for X-ray optics
}

\author{
Michael P. Biskach*a, Ryan S. McClelland ${ }^{a}$, Timo Saha ${ }^{b}$, William W. Zhang ${ }^{b}$ \\ aSGT, Inc. 7701 Greenbelt Road, Suite 400, Greenbelt, Maryland 20770, USA \\ bNASA Goddard Space Flight Center, Greenbelt, Maryland 20771, USA
}

\begin{abstract}
The flight mirror assemblies (FMA) for X-ray telescopes similar to that of the International X-ray Observatory (IXO) concept consist of several thousands of individual mirror segments. The size, shape, and location of these mirrors affect many characteristics of the telescope design. Mission requirements among other factors in turn restrict mirror segment parameters such as thickness, axial length, azimuthal span, and mass density. This paper provides an overview of the critical relationships relating to mirror segment size and configuration throughout the design and analysis of an X-ray mirror assembly. A computational analysis is presented in the form of ray tracing pairs of thin X-ray mirror segments of varying sizes aligned in gravity and supported using kinematic constraints with corresponding self weight distortions calculated using finite element analysis (FEA). The work in this paper may be used as a starting point for determining mirror segment sizes for X-ray missions like that of IXO and beyond.
\end{abstract}

Keywords: Segment Optics, X-ray Optics, Finite Element Modeling, IXO, Mirror Sizing

1.

INTRODUCTION

Future X-ray telescope missions hope to provide the tools necessary to enable scientists and researchers to quantify and fully explain various cosmological occurrences such as supermassive black holes and dark matter. By observing distant $\mathrm{X}$-rays, a clearer picture of how the universe has evolved to this point can be made. Local X-ray sources only indicate what has occurred most recently on the cosmological timescale. X-rays benefit from among other things the ability to penetrate dust clouds enabling observations that are not possible in other wavelengths [1].

For future X-ray telescopes to provide useful observations at such cosmological distances, the resolution and effective collecting area of the telescope must improve by several times over current X-ray observatories. One of the proposed methods currently under development at NASA Goddard Space Flight Center that hopes to combine high accuracy with large collecting area is thin slumped-glass mirror segments mounted in separate modules [2]. This segmented optics approach that will be discussed later in this paper hopes to surpass current X-ray telescope designs by providing a sub 10 arc second resolution and beyond without sacrificing effective area and adding prohibitive cost.

This paper presents a methodology that aims to optimize resolution of segmented mirror based optics while not sacrificing effective collecting area. Finite Element Analysis is performed for a set of slumped glass mirrors based on the International X-ray Observatory (IXO) concept. The aim of this paper is to provide a starting point for future analyses that will optimize mirror size based on a wider range of design variables compatible multiple X-ray mission concepts. In this light, additional contributing factors used to select mirror sizes that may affect optimization of both resolution and effective area are also discussed. To understand why such a study is necessary, it is useful to briefly review the capabilities of previous full shell optics based X-ray telescopes and introduce the proposed mirror shell segmentation approach.

*michael.biskach@nasa.gov; $\quad$ phone 301 286-1396 